\title{
Correspondence
}

\section{Reye's syndrome: diagnosis by muscle biopsy?}

Sir,

I read the paper by Shapira et al. ${ }^{1}$ with great interest as it was, to my knowledge, the first report of this condition. Although recurrent Reye's syndrome has keen reported, ${ }^{2}$ Pichichero and McCabe did not obtain liver biopsies in any episode. Shapira et $a l .^{1}$ noted the marked fat droplet infiltration in hepatocytes by electron microscopical examination but they did not mention any mitochondrial changes in their patients although they accepted the fact that primary generalised mitochondriopathy is the pathophysiological basis of Reye's syndrome. Were mitochondrial changes present in their case 3 ?

Shapira et al. ${ }^{1}$ pointed out the potential danger of bleeding in percutaneous liver biopsy, but in our experience of liver biopsies on at least 947 children ( 311 of them below age 2 years), this danger should not be exaggerated, provided the necessary precautions are taken. ${ }^{3}$

High levels of plasma lactate, alanine, and glutamine were accepted as indicating intramitochondrial decreased oxydation of pyruvate as a result of primary generalised mitochondriopathy. Although mitochondrial structural alterations appear to be similar in brain, muscle, heart, lung, and kidney, the activities of mitochondrial enzymes-such as citrate synthase, glutamic dehydrogenase, succinic dehydrogenase, pyruvate carboxylase, and pyruvate dehydrogenase-are decreased in the liver but not in the brain or muscles. ${ }^{4}$ Therefore $I$ think that morphological mitochondrial changes should te interpreted cautiously as evidence of primary generalised mitochondriopathy. Are these changes primary or secondary to carnitine depletion in cases of recurrent Reye's syndrome?5

Sinasi Ozsoylu Department of Paediatrics, Institute of Child Health, Hacettepe University, Hacettepe, Ankara, Turkey

\section{Dr Shapira and co-workers comment:}

Cases 1 and 3 underwent both liver and muscle biopsies but Case 2 had only a muscle biopsy; morphological abnormalities in the mitochondria were identical in all 3 muscle biopsies and in both liver biopsies. It should te mentioned that liver biopsies were descrited in recurrent Reye's syndrome secondary to systemic carnitine deficiency. 5

Dr Ozsoylu referred to his vast experience with 947 liver biopsies in children and said that the danger of bleeding should not be exaggerated provided the necessary precautions are taken. ${ }^{3}$ We agree with him, but in
Reye's syndrome the downhill course may often be very fast and not allow time for adequate correction of the bleeding diathesis; in such cases a muscle biopsy is useful.

The concept of a primary generalised mitochondriopathy in Reye's syndrome is based mainly on the mitochondrial morphological alterations seen simultaneously in the liver, muscle, brain, kidney, and heart. The fact that the activities of several mitochondrial enzymes (citrate synthase, glutamic dehydrogenase, pyruvate carboxylase, succinic dehydrogenase, and pyruvate dehydrogenase) were found to be abnormally low in the liver only, and were within the normal range in the muscle and brain tissues is disturbing. ${ }^{4}$ This may suggest that the liver is more affected in Reye's syndrome than the other tissues (brain, kidneys, and muscles). The cause of the morphological changes in the mitochondria in all those tissues remains unknown.

\section{References}

1 Shapira Y, Deckelbaum R, Statter M, Tennenbaum A, Aker $M$, Yarom R. Reye's syndrome: diagnosis by muscle biopsy? Arch Dis Child 1981; 56: 287-91.

2 Pichichero M E, McCabe E R B. Recurrent Reye'so syndrome. Am J Dis Child 1978; 132: 1097-9.

3 Ozsolyu S, Kocak N, Gursoy M H. Liver needle biopsyê in children. Turk J Pediatr 1980; 22 : 1-6.

4 Robinson B H, Taylor J, Cutz E, Gall D G. Reye's syndrome. Preservation of mitochondrial enzymes in brain and muscle compared with liver. Pediatr Res 1978; 12: 1045-7.

5 Chapoy P R, Angelini C, Brown W U, Stiff J E, Shug A L, Cederbaum S D. Systemic carnitine deficiency-a treatable inherited lipid-storage disease presenting as Reye's syndrome. $N$ Engl J Med 1980; 303: 1389-94.

\section{Birthweight, child abuse, and infant death attributed to accidents, poisonings, or violence}

Sir,

A recent analysis that links infant deaths that occurred in 1978 to birth records is very welcome. ${ }^{1}$ One striking feature is the association of infant death attributed to accidents, poisonings, and violence (ICD code E800-999) with birthweight. The incidence of such death was 0.31 per 1000 live births. Rates within the birthweight bands $<2000,2001-3000$, and $\geqslant 3001 \mathrm{~g}$ were $1 \cdot 03,0.47$, and 0.25 per 1000 respectively, a highly significant trend $\left(\chi^{2}=35 \cdot 1, \mathrm{df}=2, \mathrm{P}<0 \cdot 001\right)$. If these results are considered in the light of data from Cardiff ${ }^{2}$ relating to the incidence of non-accidental injury (not necessarily fatal) in children aged 5 years and below, the incidence is 2.7 per 1000 live births in the entire series, and $13 \cdot 0,3 \cdot 8$, and 